Article

\title{
Combined Use of MRI, fMRIand Cognitive Data for Alzheimer's Disease: Preliminary Results
}

\author{
Chiara Dachena ${ }^{1}$, Sergio Casu ${ }^{1}\left(\mathbb{D}\right.$, Alessandro Fanti ${ }^{1,2, *(\mathbb{C}}$, Matteo Bruno Lodi ${ }^{1, *(1)}$ and \\ Giuseppe Mazzarella ${ }^{1,2}$ (D) \\ 1 Department of Electrical and Electronic Engineering, University of Cagliari, 09123 Cagliari, Italy \\ 2 INFN-CA, Complesso Universitario di Monserrato, Cagliari 09042, Italy \\ * Correspondence: alessandro.fanti@diee.unica.it (A.F.); matteobrunolodi94@gmail.com (M.B.L.)
}

Received: 7 June 2019; Accepted: 31 July 2019; Published: 2 August 2019

\begin{abstract}
MRI can favor clinical diagnosis providing morphological and functional information of several neurological disorders. This paper deals with the problem of exploiting both data, in a combined way, to develop a tool able to support clinicians in the study and diagnosis of Alzheimer's Disease (AD). In this work, 69 subjects from the ADNI open database, 33 AD patients and 36 healthy controls, were analyzed. The possible existence of a relationship between brain structure modifications and altered functions between patients and healthy controls was investigated performing a correlation analysis on brain volume, calculated from the MRI image, the clustering coefficient, derived from fRMI acquisitions, and the Mini Mental Score Examination (MMSE). A statistically-significant correlation was found only in four ROIs after Bonferroni's correction. The correlation analysis alone was still not sufficient to provide a reliable and powerful clinical tool in $\mathrm{AD}$ diagnosis however. Therefore, a machine learning strategy was studied by training a set of support vector machine classifiers comparing different features. The use of a unimodal approach led to unsatisfactory results, whereas the multimodal approach, i.e., the synergistic combination of MRI, fMRI, and MMSE features, resulted in an accuracy of $95.65 \%$, a specificity of $97.22 \%$, and a sensibility of $93.93 \%$.
\end{abstract}

Keywords: Alzheimer's disease; biomedical image processing; biomedical signal processing; cognitive science; correlation analysis; graph theory; magnetic resonance imaging; medical diagnostic imaging; SVM

\section{Introduction}

Magnetic Resonance Imaging (MRI) and functional Magnetic Resonance Imaging (fMRI) of the brain are popular methods of inquiry in neuroscience [1]. These techniques are exploited to obtain important morphological and functional information in a non-invasive way [2]. MRI can probe the structural environment, whilst fMRI can be employed to detect changes in the Blood Oxygenation Level-Dependent (BOLD) signal [3]. One of the most important limitations in MRI is the Signal-to-Noise Ratio (SNR) [3], which is influenced by the static magnetic field $B_{0}$ and other parameters, e.g., time echo, time repetition, flip angle, slice thickness, and matrix size [4]. The adjustment of the cited parameters provides a good image reconstruction with a high SNR. The imaging modalities, such as computed tomography and MRI, ensure good tissue contrast and spatial resolution [2,5], which favor an adequate tissue segmentation. In fact, the possibility of detecting structural abnormalities depends on the segmentation accuracy, which in turn may improve the diseases' diagnosis [6-8].

The segmentation can be performed manually or automatically with open source brain imaging software, e.g., SPM (fil.ion.ucl.ac.uk), FSL (fmrib.ox.ac.uk), or FreeSurfer (surfer.nmr.mgh.harvard.edu). By means of these software programs, it is possible to recognize and to extract the data of the brain 
features to investigate, such as the Grey Matter Volume (GMV). Typically, in clinical practice, the GMV is quantified to assess the degree of cerebral atrophy and to monitor the neuropathologies' state [9].

One of the most prevalent neurodegenerative disorders is Alzheimer's Disease (AD). This pathology is characterized by a severe derangement of cognitive functions and structural changes within the brain. The pathological hallmarks are the accumulation of $\beta$-amyloid (A $\beta 1-42)$ plaques and tau tangles, which cause cognitive and structural damage [10]. Several studies exploited fMRI to diagnose neurological pathology, such as schizophrenia [11], AD, and Mild Cognitive Impairment (MDI) [12]. In functional imaging, the BOLD signal reflects the activity degree of brain areas and can be used to analyze maps of functional connectivity or network measures [3]. The most important issue of fMRI is the physiological noise, which derives from cardiac, respiratory and head movements. In fact, BOLD signal can be corrupted by this kind of noise since it fluctuates within the same frequency band [13,14]. In commercial software for brain imaging, there are several automatic functionalities to remove these artifacts, which can alter the BOLD signal and its relevance, such as the Component-based method (CompCor) [15].

New approaches founded on graph theory $[16,17]$ have been proposed to investigate in vivo alterations in functional and structural cerebral networks by means of fMRI. Several studies have shown that graph theory is sensitive in providing network measurements of psychiatric and neurological disease, as in AD diagnosis [18,19].

In the literature, several research groups have focused on the use of MRI and fMRI to identify and stress the differences between AD and healthy subjects. Some work from the literature studied exclusively the anatomical structure, to highlight volume brain modifications $[5,20]$, whereas others analyzed only functional aspect to assess the cognitive status of the patients [21,22]. Other studies showed the feasible correlation between these two aspects [23,24], but using other types of measurements. To the best of the author's knowledge, none of the cited work ever carried out an investigation about the possible correlation between the ROI volumes, retrieved from MRI, and the graph connectivity measurements, derived from fMRI acquisitions.

Besides the pure statistical approach, from the meta-analysis of the literature, it is possible to identify the trend of employing machine learning to classify AD patients from HCs. On the one hand, many of them used only structural information obtained from MRI images [25,26]; whilst, on the other hand, others used both structural and functional information to train the classifier [27,28]. The cited works faced the problem of recognizing diseased subjects using a reduced number of features, which can be derived by a single clinical exam. It should be pointed out that the functional information was derived from PET exams. None of them used graph theory to extract functional information of fMRI, but pulled out other types of information from fluorodeoxyglucose positron emission tomography (FDG-PET). Moreover, relying on the assumption, shared by the imaging community, that given a high number of features, a classifier would perform better, this work also investigates the combined use of the MRI- and fMRI-derived features, together with the Mini Mental State Examination (MMSE), to investigate differences between AD and HC subjects. In this work, the MMSE is employed instead of other clinical indexes, such as the Neuropsychiatric Inventor (NPI-Q) [29] or the Clinical Dementia Rating (CDR) [30]. The chosen cognitive screen is known to suffer from unstable inter-rater reliability and from a limited score range; however, it is free, widely available, quick to administer, and allows experts and non-experts to measure the general cognitive ability, while allowing the comparison between different studies [31].

In this paper, the data were extracted from the Alzheimer's Disease Neuroimaging Initiative (ADNI) database, consisting of $33 \mathrm{AD}$ patients and 36 healthy controls. The MRI data were processed with the Statistical Parametric Map (SPM) segmentation software to extract the structural feature, while the BOLD signal from fMRI exams was exploited to evaluate connectivity measures through the graph theory. The synthesis of the findings derived from MRI and fMRI data processing, through Spearman correlation analysis between the Clustering Coefficient (CC) and GMV, can provide clinicians relevant 
clinical information about AD diagnosis. Moreover, for the first time, to the best of our knowledge, we evaluated the possibility of correlating the GMV with the CC.

Finally, the measurements from three data, i.e., MRI, fMRI, and MMSE, were combined to discriminate between AD and HC, through a Support Vector Machine (SVM). The findings presented in this work demonstrate that, instead of exploiting one type of measurement, using the combined and synergistic information from different imaging techniques (i.e., $\mathrm{fMRI}$ and MRI) and the clinical data from MMSE enhanced the AD classification, with an accuracy of $95.65 \%$, a sensitivity of $93.93 \%$, and a specificity of $97.22 \%$. In fact, with a unimodal classification method, it is possible to obtain an accuracy of $62.32 \%$, a sensitivity of $66.66 \%$, and a specificity of $58.33 \%$ for fMRI; and an accuracy of $50.72 \%$, a sensitivity of $30 \%$, and a specificity of $69.44 \%$ for MRI.

\section{Methods and Procedure}

\subsection{Magnetic Resonance Imaging}

The need to extract biological information from intact biological systems using MRI and fMRI introduced new technologies that allowed the study of human brain activity with greater detail and resolution [32]. MRI offers more tissue contrast possibility than other radiological methodologies, such as computerized tomography. This is possible by properly setting the time in which the Radiofrequency (RF) impulsive excitement is produced and its derived signal is registered. The contrast helps in discriminating different biological tissues and structures. However, it depends on both the longitudinal relax time $\left(\mathrm{T}_{1}\right)$ and transversal relax time $\left(\mathrm{T}_{2}^{*}\right)[32]\left(\mathrm{T}_{1}\right.$ is the time necessary to recover $63 \%$ of the longitudinal magnetization present before the RF impulse $[4,32]$ ).

In MRI, high $\mathrm{T}_{1}$ is necessary to distinguish white matter, gray matter, and cerebrospinal liquid. $T_{2}$ is the time required for the transversal magnetization vector to decrease at $37 \%$ of the maximum of its intensity [4]. In MRI, low $\mathrm{T}_{2}$ are necessary to distinguish the three above-cited cerebral tissues [4]. As a matter of fact, to perform studies on AD using MRI images, data acquired with high $T_{1}$ and low $T_{2}$ are necessary [4]. With these settings, the structural features can be adequately evaluated [4,32].

The MRI possibilities are often limited by the achievable SNR and the Contrast-to-Noise Ratio (CNR). Both the SNR and CNR have been shown to increase with the static magnetic field strength $B_{0}$ [32]. Besides the influence of the magnetic field $B_{0}$, the SNR is affected by other parameters, such as Time Echo (TE), Time Repetition (TR), Flip Angle (FA), slice thickness, and matrix size [4,32]. The work of Coriasco et al. [4] contained further details about these parameters and their influence on SNR. The intensity of the magnetic resonance signal is defined by the tissue volume. Indeed, high tissue volumes produce an intense MR signal, which in turn increases the SNR. However, this MRI measurement involves the so-called partial volume effect, which consequently lowers the image resolution levels [33]. This is a relevant aspects in the elaboration of MRI images. In fact, the poorer SNR of thin slices can be compensated to some extent by increasing the number of acquisitions or by a longer TR [34]. Considering the work of Pohmann et al. [35], the SNR strictly depends on $\mathrm{T}_{1}, \mathrm{~T}_{2}^{*}$, TR, TE, FA, and the sensitivity of the receiver coil.

Since this work made use of morphological data arising from MRI, the influence of all the cited parameters $[4,32,35]$ was critically considered when patients and images to analyze were selected.

\subsection{Magnetic Mechanism of the BOLD Signal}

The BOLD signal represents the hemodynamic response of cerebral activation [36]. Several studies demonstrated that BOLD signal alterations represent a complex interplay between changes in the metabolic rate of cerebral blood volume, blood flow, and oxygen consumption rate [1,36]. In fact, the increased neuronal activity induces modifications in local vasculature and thus causes changes in the deoxyhemoglobin $(\mathrm{dHB})$ concentration [36].

The $\mathrm{dHB}$ and the oxyhemoglobin present paramagnetic and diamagnetic behavior, respectively, leading to a shift of the local magnetic susceptibility [1]. In MRI, the material magnetization is directly 
proportional to the strength of the magnetic field [4]; hence, different magnetic responses provoke different signals in MRI. Logothetis [3] demonstrated that the $\mathrm{dHB}$ induces field inhomogeneity and neural activity, resulting in BOLD signal reduction.

The importance of the temporal preprocessing step for the BOLD signal was underlined in [37]. In our study, preprocessing steps are implemented using the CONNtoolbox. This allows specifying possible temporal confounding factors, such as movement parameters and artifacts, and BOLD signals obtained from noisy ROIs, such as White Matter (WM) and Cerebrospinal Fluid (CSF). Each temporal confounding factor $c_{n}(t)$ is then regressed from the BOLD signal, called $\operatorname{BOLD}(v, t)$, at each voxel as follows [37]:

$$
B O L D(v, t)=B O L D *(v, t)-\sum_{n=1}^{N} a_{n}(v) \cdot c_{n}(t)-\sum_{k=1}^{K} \sum_{n=1}^{M_{k}} b_{k n}(v) \cdot d_{k n}(t)
$$

where $B O L D *(v, t)$ is the observed bold signal at voxel $v$ and time $t$, while $N$ is the number of temporal confounds, and $\mathrm{d}_{k}(t)$ are confound s from $\mathrm{K}$ noise ROIs, each characterized by $\mathrm{M}_{k}$ components of the magnetization signal from each noise ROI.

The resulting residual BOLD signals are band-pass filtered $(0.001 \mathrm{~Hz}-0.01 \mathrm{~Hz})$. Mathematical details are shown in Whitfield-Gabrieli et al. [37].

\subsection{Data Acquisition and Preprocessing}

All subjects used in this study were selected from the Alzheimer's Disease Neuroimaging Initiative (ADNI) database. Data were selected based on the availability of the resting-state fMRI dataset for age-matched healthy normal subjects and AD patients. A total of 69 subjects, 33 Alzheimer's disease (18 females, 15 males, age $\pm S D=72.72 \pm 7.52$ years, mini-mental state $=22.6667 \pm 2.45$, with a median value of 22) and 36 healthy controls (21 females, 15 males, age $\pm \mathrm{SD}=72.51 \pm 5.40$ years, mini-mental state $=26.6667 \pm 1.36$, with a median value of 29), of the ADNI-2 database were considered. For each subject, only one acquisition was employed. The first acquisition of each subject was used, resulting in a total of 69 acquisitions. Subjects' demographic data are summarized in Table 1.

This database includes more subjects (around one thousand). However, not every subject presents both morphological and functional images. Furthermore, some patients in the database may be initially classified as AD (or HC), and then, their clinical status is changed. Those patients were excluded from our study, i.e., we employed subjects that did not change their ADNI classification. Moreover, a subject can present more MRI and/or fMRI acquisitions taken at different time steps. The data used in this study were the only that presented contemporary anatomical MRI, functional MRI, and MMSE for each acquisition in the ADNI database. The MMSE feature was employed in this study because it is an accurate indicator of the clinical status and since it is widely used in clinical practice and largely available for most of the subjects included in the ADNI database. Not all subjects from the ADNI database presented the NPI-Q and/or the CDR indexes [29,30]. Additionally, as regards the Mild Cognitive Impairment (MCI) subjects contained in the database, it should be noted that they present only anatomical MRI or fMRI data, and for this reason, we did not include them in our study. Moreover, in this work, images acquired with the same machine model and sequences were used to avoid problems caused from using different apparatuses and to overcame bias, as well as other problems due to different acquisition methods [4,32,35].

A major claim related to the number of subjects used in this study (i.e., 69) could be that the dataset dimension is not sufficient for both statistical analysis and the employment of a supervised approach for patients' classification. However, from the current available literature, studies such as $[23,38]$ have used a number of subjects between 19 and 28 to investigate the differences between $A D$ patients and healthy subjects. Therefore, the use of a dataset 2.5-times larger should be regarded as an added value. On the other hand, studies make use of a spread and wide dataset (max. six hundreds patients) to perform statistical analysis, investigate the pathology using the graph theory or rely on 
automatic classification algorithms [26,39,40], and have employed a single imaging technique [41,42], i.e., or MRI or fMRI or PET were used, but never combined together. Moreover, many of them specify the conditions used for all subjects, which would surely be different, thus probably leading to a bias in their results. Our paper has the aim of establishing the effectiveness of analyzing morphological, functional, and clinical features in AD subjects in a synergistic way using a reliable dataset.

Resting state data and structural MRI scans were acquired with a 3.0 T Philips Medical System scanner. The acquisitions were performed by ADNI according to its acquisition protocol [26]. The MRI data included high-resolution $T_{1}$-weighted scans and an 8-channel receive-only head coil. The parameters included: sagittal plane, slices $=170, \mathrm{TR}=6.77 \mathrm{~ms}, \mathrm{TE}=3.13 \mathrm{~ms}, \mathrm{TI}=0 \mathrm{~ms}, \mathrm{FA}=9^{\circ}$, matrix $=256 \times 256$, and voxel size $=1.0 \times 1.0 \times 1.2 \mathrm{~mm}^{3}$. All the resting state scans consisted of 140 functional volumes and were obtained while participants were resting in the scanner with eyes open. The parameters included: $\mathrm{TR}=3000 \mathrm{~ms}, \mathrm{TE}=30 \mathrm{~ms}$, flip angle $=80^{\circ}$, slice thickness $=3.313 \mathrm{~mm}$, and 48 slices. Resting state images and structural scans were elaborated in Nifty format [43].

Table 1. Demographic and neuropsychological characteristics of the subjects.

\begin{tabular}{cccc}
\hline & HC Subjects & AD Patients & $p$-Value \\
\hline Number of subjects & $36(21 \mathrm{~F} / 15 \mathrm{M})$ & $33(18 \mathrm{~F} / 15 \mathrm{M})$ & $p>0.99^{1}$ \\
Age, years & $72.51( \pm 5.40)$ & $72.72( \pm 7.52)$ & $p>0.05^{2}$ \\
MMSE & $26.6667( \pm 1.36)$ & $22.6667( \pm 2.45)$ & $p=2.828 \times 10^{-123}$ \\
\hline
\end{tabular}

${ }^{1}$ The $p$-value was obtained by the Pearson $\chi^{2}$ two-tailed test; ${ }^{2}$ the $p$-value was obtained by a two-sample two-tailed $t$-test; ${ }^{3}$ the $p$ value was obtained by the Pearson $\chi^{2}$ two-tailed test.

The images were processed using the CONN functional connectivity toolbox v15.a (nitrc.org, Version 02142, Cambridge, MA, USA). This software exploits SPM routines to perform structural and functional analysis. The preprocessing procedures are summarized in Figure 1. The first step was to realign the MRI structural data to undo the effects of subject movements during the scanning session. The differences between each successive scan and a reference scan, which is the average of all scans in the time series, was minimized [44].

Afterwards, the data were transformed using linear warps into a standard anatomical space, the Montreal Neurological Institute (MNI) space [45]. A slice timing correction step was necessary to compensate for slice acquisition delays, which may add up to significant temporal shifts between the expected and the actually measured BOLD signal [46]. Finally, it was useful to co-register (to realign the $256 \times 256 \mathrm{MRI}$ images with the $64 \times 64 \mathrm{fMRI}$ images to work in a unique reference frame) functional and anatomical images [47]. We also included a scrubbing step for both motion artifacts and outliers in fMRI images. The Artifact Detection Tools (ART) software (nitrc.org) was used to identify scans affected by movement-related artifacts. Then, functional images were smoothed using a Gaussian kernel of $8 \mathrm{~mm}$ FWHM. The movements and the physiological noise were removed by linear regression. More precisely, the noise source considered was the signals from white matter and cerebrospinal fluid, the six head motion parameters, and the outlier scans detected during the scrubbing process [37]. Then, the preprocessed images were bandpass filtered to $0.001 \mathrm{~Hz}-0.01 \mathrm{~Hz}$ to reduce the noise. 


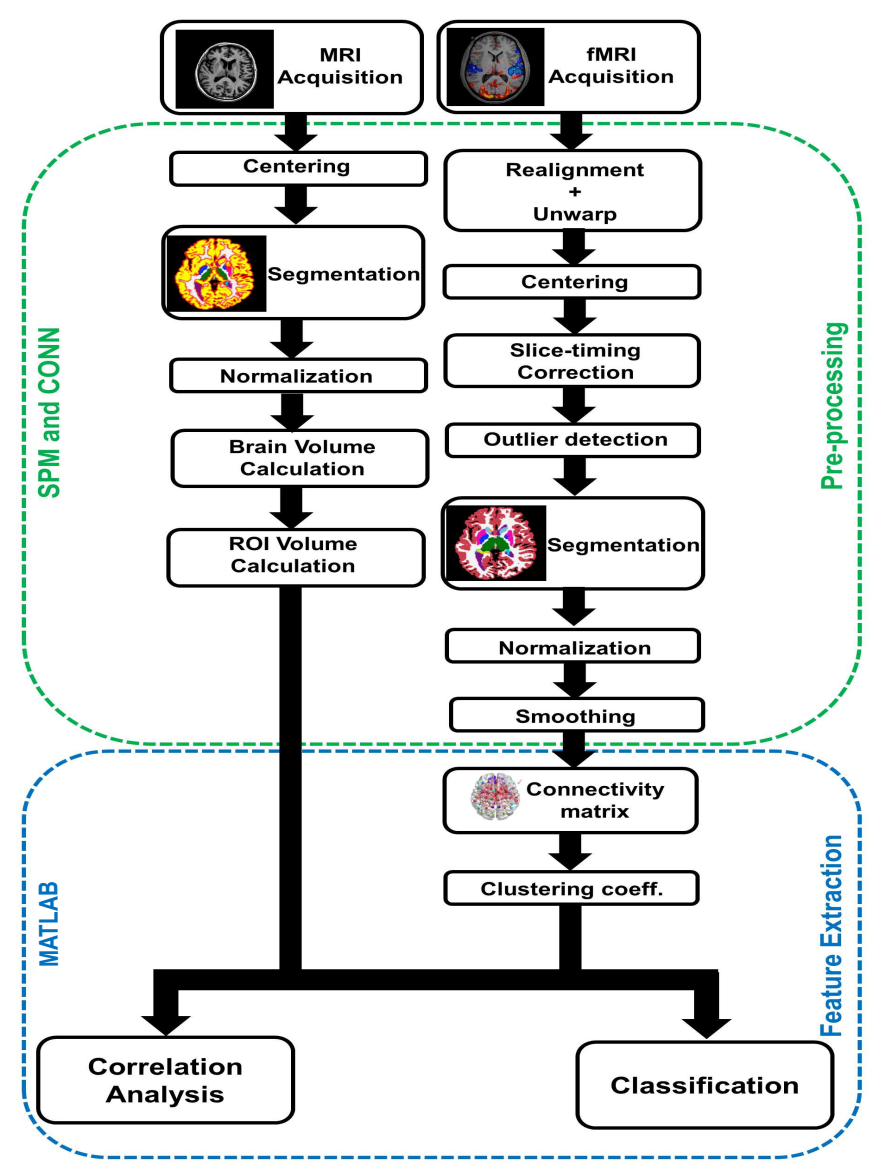

Figure 1. Preprocessing and elaboration steps for MRI and fMRI images.

\subsection{Image Segmentation with SPM}

The segmentation is a process in which the image is divided into homogeneous and non-overlapping regions, where pixels or voxels have similar attributes such as intensity or color [48]. In MRI brain analysis, brain tissue should be classified into three tissue types, namely GM, WM, and CSF [48]. This distinction can be performed employing several different methods, e.g., manual segmentation, intensity-based methods, atlas-based methods, surface-based methods, and hybrid segmentation methods [48]. However, none of them is universally accepted in literature.

Therefore, in this study, the SPM automatic intensity-based Gaussian segmentation was employed to recognize brain structures of interest and to use them for further analysis. In this method, each tissue class is modeled by a Gaussian distribution [48] and the histograms of brain MRI images have three main peaks corresponding to the three main tissue classes. These three peaks allow distinguishing the brain tissues and performing the correct operation of segmentation. This approach uses a "modified Gaussian Mixture Model" (GMM) [49], which is a standard technique widely used by many tissue classification algorithms [50]. The main advantages of the standard GMM are the easy implementation and the small number of parameters that can be efficiently estimated $[49,50]$.

\subsection{Brain Volume Calculation and Mask Construction}

After the segmentation, the brain tissue images were used to quantify the whole brain volume and the intracranial volume. The whole brain volume was calculated as the sum of the volume of GM and WM; whereas the intracranial volume was calculated as the sum of GM, WM, and CSF. 
The total brain volume was normalized for the whole intracranial volume to eliminate gender disparity [51]:

$$
V_{n}=\frac{W M+G M}{W M+G M+C S F}
$$

In fact, as can be seen in Figure 2a,c, males and females presented different total brain volumes $(p<0.05)$. After normalization, such a difference fell, as shown in Figure $2 \mathrm{~b}, \mathrm{~d}(p>0.05)$. These results strongly agreed with those found in [52]. Afterwards, employing the Wake Forest PickAtlas software (fmri.wfubmc.edu) [53], the masks were limited to a priori Regions Of Interest (ROIs) through the AAL (Automated Anatomical Labeling) atlas [45]. This is one of the possible atlases to be used to extract masks, and it is often employed for studies involving Alzheimer's and MRI [54,55] or fMRI [56]. This atlas includes 90 ROIs (45 per hemisphere). These masks were employed to quantify ROI volume and connectivity as in $[54,55]$. The whole brain, intracranial, and ROIs volume were determined with the get_totals script (cs.ucl.ac.uk), such as in [57,58]. This script takes one segmented MRI set, i.e., all the images for a given brain tissue, as input and then returns its volume expressed in $\mathrm{mm}^{3}$. Furthermore, to quantify ROI volume, the script takes the GM image and the ROI mask, and then, it calculates the intersection between these images, finally returning its volume in $\mathrm{mm}^{3}$.
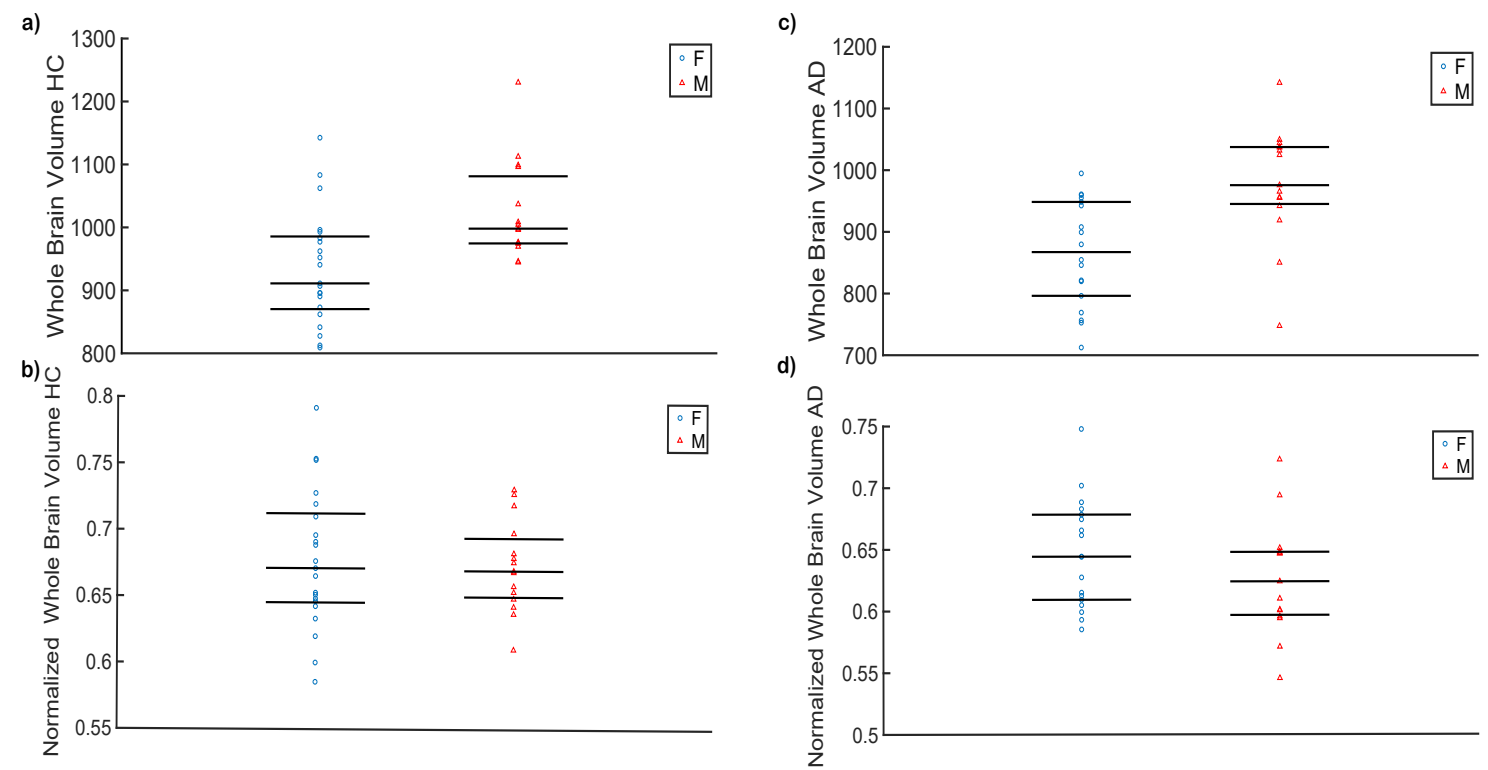

Figure 2. Statistical differences in whole brain volume between male and female subjects for the HC group (a) and AD group (c) $(p<0.05)$. After whole brain normalization $\left(V_{n}\right)$, the scatter plots show a similar range of variation for both HC $(\mathbf{b})$ and ADs $(\mathbf{d})$ subjects $(p>0.05)$.

\subsection{Connectivity Matrix and Network Measure}

As stated in Section 2.2, the BOLD signals were extracted from fMRI images. Each signal was used to create connectivity matrices of $90 \times 90$, in which each row and each column stood for one ROI of the atlas. Each value in the matrices represented the functional connectivity. The functional connectivity is defined as the statistical dependencies among remote neurophysiological events [36,59], and it is measured with Pearson's correlation coefficient between two BOLD signals, mathematically [60]:

$$
r=\frac{\sigma_{p q}}{\sigma_{p} \sigma_{q}}
$$

where $\sigma_{p q}$ is the covariance of the two BOLD signals, and $\sigma_{p}$ and $\sigma_{q}$ are the standard deviations of the $p$ th and $q$ th signal, respectively. The connectivity matrices, shown in Figure 3, were used to perform the network measure, i.e., the calculation of a clustering coefficient. 


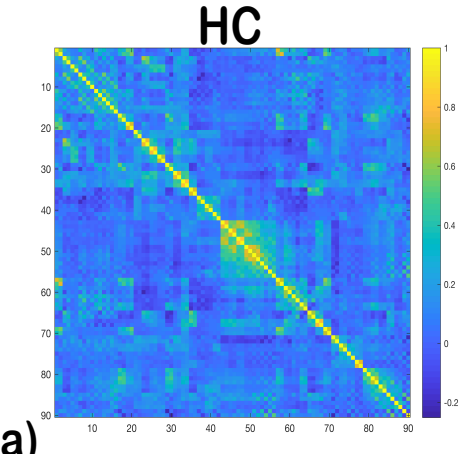

a)

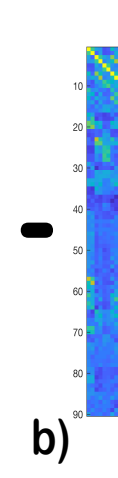

AD

Figure 3. Connectivity matrices for the (a) AD group, (b) HC group, and (c) the difference HC-AD.

Other studies [61,62] demonstrated that this parameter is useful to discriminate the patient from the healthy control in Alzheimer's disease. Similarly to [61], the clustering coefficient was used with another graph measure, path length, to evaluate the global organization of large-scale networks or the small world index and then evaluate the AD progression [63]. In our study, the clustering coefficient was used in combination with the relevant morphological brain feature to estimate the possible correlation between functional and morphological information in AD patients and to discriminate healthy controls from patients.

These matrices showed the mean functional connectivity in the AD group (Figure 3a), in HC subjects (Figure $3 b$ ), and the difference between the HC and AD groups (Figure 3c). Figure $3 c$ displays that there was a difference between the means of the correlation coefficients. This difference arose for several ROIs, but not for all of them. The CC of a vertex represents the likelihood that its neighbors are connected [64]. The clustering coefficient $C_{i}$ of a vertex $i$ with degree $k_{i}$ is usually defined as the ratio of the number of existing edges $\left(e_{i}\right)$ between the neighbors of $i$, and the maximum possible number of edges between the neighbors of $i[16]$ :

$$
C_{i}=\frac{2 e_{i}}{k_{i}\left(k_{i}-1\right)}
$$

This measurement was calculated with the Brain Connectivity Toolbox (n-connectivity-toolboox.net).

\subsection{Statistical Analysis}

All statistical analyses in this study were performed using the MATLAB Statistic Toolbox. In order to test if the normalized volume and GMV were significantly different among the HC and AD groups, a Mann-Whitney U-test was performed. When multiple comparisons are made between data, false positives ought to be thrown back through appropriate corrections, such as Bonferroni's correction [65] or the False Discovery Rate (FDR) [66]. In this analysis step, only Bonferroni's correction was performed because it is more conservative [28]. Finally, to test the correlation between MMSE and GMV and between CC and GMV, Spearman's correlation was employed.

\subsection{Support Vector Machine Classifier}

As discussed in the Introduction, to help the clinicians to investigate the AD status, the use of machine learning has been explored in the literature [27,28]. Among the available supervised approaches, Support Vector Machine (SVM) is an efficient supervised learning method, which can organize large dimensional data classification [67]. This linear classifier is very efficient in working with two-class linearly-separable tasks, i.e., with data of the binary class [67]. The classification operates finding the hyperplane with the maximum possible margin between the two classes.

The capability and usefulness of the SVM classification method to discriminate AD patients from healthy ones was demonstrated in other studies $[27,28,68]$. However, it must be stressed that 
these works made use of structural brain features only, i.e., morphological information derived from MRI [68]. Even when a reduced number of feature is employed, this method should be regarded as a partial and limited approach to the development of a reliable diagnostic tool.

In contrast with other studies, which used different imaging modalities, such as FDG-PET [27] or investigated CSF [28], the approach proposed in this paper exploits fMRI. In these studies [27,28], which presented both anatomical and another type of functional images, the number of subjects was comparable to the one used therein. In fact, in other studies that used MRI, such as [26,39,41], subjects presented only morphological images. Another study used only fMRI acquisition, presenting 40 subjects, $20 \mathrm{ADs}$ and $20 \mathrm{HCs}$ [42]. All these considerations allowed us to say that our number of subjects was sufficient for this type of analysis. The approach proposed in our paper may be of interest for other researchers, which could access a larger dataset.

To train the SVM classifier, we used the leave-one-out method, as in [27,42]. In this case, the analysis was repeated for each subject: in every iteration, one sample was left out to test the classifier, and the remaining subjects were fed to the classifier for training [69]. The procedure was repeated until all subjects were used at least once as the test sample. In this study, the SVM classifier was implemented in MATLAB.

Three different SVM classifiers were tested. Firstly, the unimodal approach was assessed. Therefore, two different SVM systems were trained, one using the MRI measurements and the other one employing the fMRI-derived feature. Then, the MRI, fMRI, and MMSE information was combined to train a multimodal SVM classifier. The MMSE indicator was strongly correlated with the pathology and thus could favor the investigation between HC and AD subjects [29,31,70].

\section{Results}

The data used in this study were obtained from the ADNI open database, for a total of 69 acquisitions of $\mathrm{AD}$ and $\mathrm{HC}$ subjects. The goal of this paper was to compare $\mathrm{AD}$ patients with a group of $\mathrm{HC}$ to evaluate their morphological and functional differences, both through the correlation analysis and through the SVM classification.

First, a Mann-Whitney U-test was performed to explore the statistical differences of the normalized whole brain volume between the AD patient group and the control group [71]. In Figure 4, the results show that there was a significant difference between the two groups $(p=0.0011, z=-3.26)$. Moreover, AD patients presented a lower volume when compared with HCs. The findings from $[5,20]$ supported our results and suggested that $\mathrm{AD}$ is related to a lower brain volume with quantifiable differences with respect to the HC subjects. 


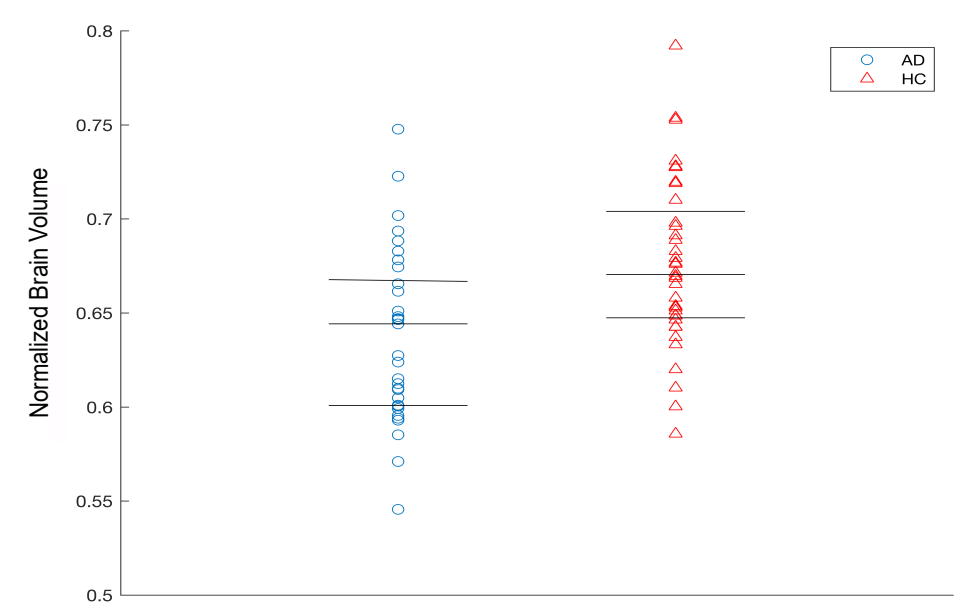

Figure 4. Statistical differences in normalized whole brain volume between HC and AD groups $(p<0.05)$.

Furthermore, 36 ROIs presented a lower GMV $(p<0.05)$ in AD patients compared with HC. Even in this case, a Mann-Whitney U-test was performed. After Bonferroni's statistical correction, only two ROIs survived, the right rolandic operculum and the left superior temporal gyrus. Given the AAL atlas, some of the regions that did not survive after Bonferroni's correction (the discarded 34 ROIs) belonged to frontal lobe, as is reported in Table 2, which is involved in cognitive function. Supposedly, these ROIs are affected by the pathology state [72]. Moreover, many of these regions were found in other structural studies that used the ADNI database, such as insula [38], frontal cortex [38,73], posterior cingulate cortex [23,73,74], hippocampus [73,75], fusiform [76], temporal lobe [38,73], and, in particular, left middle temporal gyrus $[45,76]$. These confirm and validate our results.

As performed in other studies [76,77], the possible correlation between GMV and the cognitive status, i.e., MMSE, across groups was studied. The AD patients had a MMSE score [78] of $12 \div 28$, whereas HC had an MMSE score of $24 \div 30$. This analysis step was necessary to evaluate if cognitive status was associated with structural status. Spearman's correlation coefficient was used [79]. Across groups, GMV was correlated to the MMSE score in 10 ROIs, as we can see in Table 3, but after Bonferroni's correction, no one survived. Even in this small-sized database, AD patients were associated with lower values of MMSE, with respect to the HC subjects. The lower score of MMSE was associated with a lower GMV in the left superior temporal gyrus $(\rho=0.3602, p=0.0024)$, as shown in Figure 5. This result shows that in AD subjects, the cognitive status was positively correlated with GM atrophy. Thus, for the atlas and the dataset analyzed in this study, this finding probably implies that a lower cognitive capacity is linked to a lower brain volume, which is the first consequence of pathology in $\mathrm{AD}[80]$. 
Table 2. Regions with a significant difference in grey matter volume between AD and HC that did not survive after Bonferroni's correction.

\begin{tabular}{ccc}
\hline Region Number & Location & $p$-Value \\
\hline 1 & Precentral L & 0.0259 \\
2 & Precentral R & 0.0093 \\
4 & Frontal Sup R & 0.0275 \\
5 & FrontlSupOrbL & 0.0275 \\
8 & Frontl Mid R & 0.0371 \\
11 & Frontl InfOperL & 0.0061 \\
12 & Frontl Inf Oper R & 0.000631 \\
14 & Frontl Inf TriR & 0.0251 \\
17 & Rolandic Oper L & 0.0011 \\
29 & Insula L & 0.013 \\
30 & Insular R & $0.00 \mathrm{e}-34$ \\
37 & Hippocampus L & 0.0087 \\
43 & Calcarine L & 0.045 \\
44 & Calcarine R & 0.0361 \\
47 & Lingual L & 0.0214 \\
48 & Lingual R & 0.0123 \\
50 & Occipital Sup R & 0.0468 \\
56 & Fusiform R & 0.0054 \\
57 & Postcentral L & 0.0251 \\
58 & Postcentral R & 0.0063 \\
59 & Parietal SupL & 0.0495 \\
60 & Parietal Sup R & 0.0315 \\
61 & Parietal Inf L & 0.0284 \\
62 & Parietal Inf R & 0.0093 \\
63 & Supramarginal L & 0.0160 \\
64 & Supramarginal R & 0.0115 \\
65 & Angular L & 0.0166 \\
66 & Angular R & 0.009 \\
67 & Precuneus L & 0.010 \\
68 & Precuneus R & 0.0131 \\
73 & Putamen L & 0.0442 \\
75 & Pallidum L & 0.0040 \\
76 & Pallidum R & 0.0394 \\
82 & Temporal Sup R & 0.0023 \\
\hline & & \\
\hline 4 & & \\
5 & &
\end{tabular}

Table 3. Regions with significant correlation between grey matter volume and MMSE across AD and HC groups.

\begin{tabular}{cccc}
\hline Region Number & Location & $\rho$ & $p$-Value \\
\hline 11 & Frontl Inf Oper L & 0.2460 & 0.0416 \\
12 & Frontl Inf Oper R & 0.27533 & 0.0221 \\
17 & Rolandic Oper L & 0.2618 & 0.0298 \\
18 & Rolandic Oper R & 0.3453 & 0.0037 \\
29 & Insula L & 0.2455 & 0.0421 \\
37 & Hippocampus L & 0.2783 & 0.0206 \\
67 & Precuneus L & 0.261 & 0.0309 \\
73 & Putamen L & 0.2601 & 0.0309 \\
75 & Pallidum L & 0.2632 & 0.0289 \\
\hline
\end{tabular}




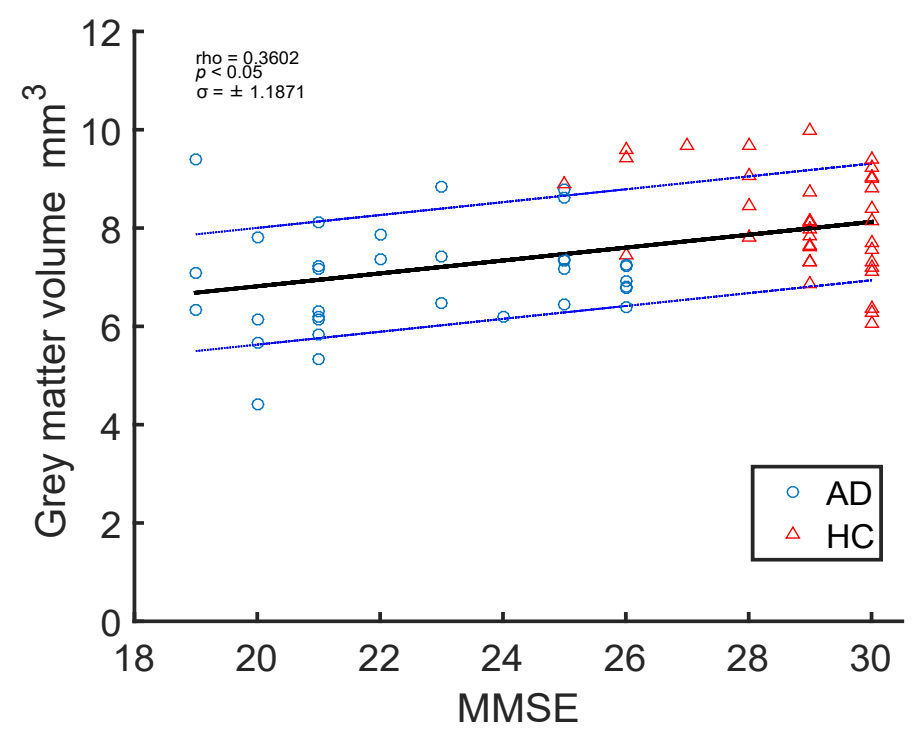

Figure 5. Correlation between GMV and MMSE within groups in the left superior temporal gyrus.

Successively, the correlation analysis between GMV and the clustering coefficient in the ROIs was assessed. This step was necessary to evaluate if any possible correlation between structural and functional information existed and can be exploited as a clinical feature to identify a possible difference between the $\mathrm{HC}$ and $\mathrm{AD}$ groups. The analysis was performed considering the two groups separated and then combining $\mathrm{AD}$ and $\mathrm{HC}$ subjects. This choice was aimed to identify if the correlation coefficient, $\rho$, can be statistically significant for a given ROI and to understand in which measure it can contribute to the knowledge of the differences between healthy control and diseased subjects, also when the two classes are grouped and mixed. In the literature, nobody has demonstrated this possible relationship using structural MRI and fMRI. Previous studies employed GMV as a covariate $[23,73,74,81]$ or evaluated the anatomical overlap between GM atrophy and functional disconnection [80].

Other techniques, such as diffusion MRI [24], were used to evaluate such a probable correlation. In this work, across groups, the GMV and the CC were correlated in seven ROIs; after Bonferroni's correction, no ROI survived. From Figure 6a, higher GMV was associated with high CC in the right supplementary motor area $(\rho=0.3107, p=0.0096)$. Within the HC group, nine ROIs presented correlation between GMV and CC. Higher GMV was associated with a higher correlation coefficient in the left calcarine (rho $=0.4597, p=0.0052$ ), as shown in Figure $6 \mathrm{~b}$. Within the AD group, as observed from Figure $6 c$, only four ROIs presented a correlation between GMV and CC. Higher GMV was associated with a higher CC in the left hippocampus $(\rho=0.4014, p=0.0213)$. Other results of the correlation analysis are shown in Table 4 . The findings from Table 4, considering the HC subjects, indicated that the correlation coefficient of GMV and CC of both the supplementary motor areas and the cuneus, the left calcarine, the middle-left occipital, and left angular were statistically significant. When looking at the $\rho$ between structural and functional information for these ROIs in the AD group, it can be noticed that they did not survive the Bonferroni correction. Indeed, for the AD set, the number of ROIs that presented a strong and significant correlation between CC and GMV was lower. They were middle-left frontal, frontal inferior right operculum, left hippocampus, and left Heschl. It can be inferred that considering a homogeneous group (or HC or AD), the correlation between some ROIs was significant, but the ROIs were not the same. This can be relevant information, since a positive correlation coefficient indicates that lower values of GMV are associated with lower CC values, implying that the pathology determines differences in structural and functional features. When $\mathrm{AD}$ and $\mathrm{HC}$ subjects were grouped, it can be observed, after Bonferroni's correction, that most of the ROIs with a statistically-significant correlation coefficient were areas that were not pointed out when analyzing the other groups (left precentral, left and right angular, left paracentral lobule, and 
right putamen). However, for both the left and right supplementary motor area and the left cuneus, significant results were found, as when the HC group was analyzed. It is evident that the GMV of these ROIs can be associated with a strong relationship with the CC in healthy subject, even for AD patients.
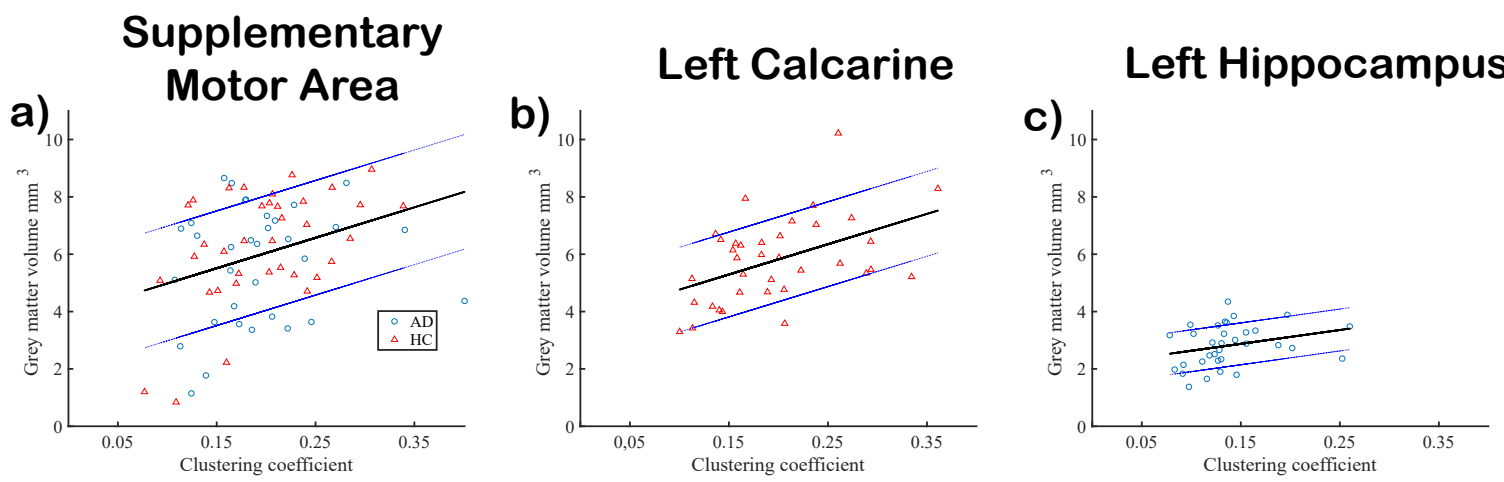

Figure 6. Correlation between the Clustering Coefficient (CC) and GMV (a) within groups in the right supplementary motor area $(\rho=0.3107, \sigma= \pm 1.998),(\mathbf{b})$ in the HC group in left calcarine $(\rho=0.4597$, $\sigma= \pm 0.4787$ ), and (c) in the AD group in left hippocampus $(\rho=0.4014, \sigma= \pm 0.7299)$. The three regions with the highest correlation values are presented.

Table 4. Regions with significant correlation between Grey Matter Volume (GMV) and the Clustering Coefficient (CC) across and with AD and HC groups. The area that survived Bonferroni's correction is highlighted in bold font.

\begin{tabular}{ccccccc}
\hline \multirow{2}{*}{ ROI-Number and Description } & \multicolumn{2}{c}{ HC + AD } & \multicolumn{2}{c}{ HC } & \multicolumn{2}{c}{ AD } \\
& $\boldsymbol{\rho}$ & $\boldsymbol{p}$-Value & $\boldsymbol{\rho}$ & $\boldsymbol{p}$-Value & $\boldsymbol{\rho}$ & $\boldsymbol{p}$-Value \\
\hline 1 Precentral L & $\mathbf{0 . 2 4 1 7}$ & $\mathbf{0 . 0 4 5 7}$ & 0.2981 & 0.0778 & 0.1340 & 0.4556 \\
7 Frontal Mid L & 0.2054 & 0.0904 & 0.0996 & 0.5619 & $\mathbf{0 . 3 6 6 3}$ & $\mathbf{0 . 0 3 6 7}$ \\
12 Frontl Inf Oper R & 0.2327 & 0.0545 & 0.0965 & 0.5742 & $\mathbf{0 . 3 7 6 7}$ & $\mathbf{0 . 0 3 1 4}$ \\
19 Supp Motor Area L & $\mathbf{0 . 2 4 3 5}$ & $\mathbf{0 . 0 4 4 0}$ & $\mathbf{0 . 3 6 2 9}$ & $\mathbf{0 . 0 1 7 5}$ & 0.0775 & 0.6669 \\
20 Supp Motor Area R & $\mathbf{0 . 3 1 0 7}$ & $\mathbf{0 . 0 0 9 6}$ & $\mathbf{0 . 3 9 5 9}$ & $\mathbf{0 . 0 1 7 5}$ & 0.1942 & 0.2777 \\
37 L Hippocampus & 0.4014 & 0.0213 & -0.0337 & 0.8450 & $\mathbf{0 . 4 0 1 4}$ & $\mathbf{0 . 0 2 1 3}$ \\
43 Calcarine L & 0.1828 & 0.1326 & $\mathbf{0 . 4 5 9 7}$ & $\mathbf{0 . 0 0 5 2}$ & -0.1334 & 0.4578 \\
45 Cuneus L & $\mathbf{0 . 2 5 7 8}$ & $\mathbf{0 . 0 3 2 7}$ & $\mathbf{0 . 4 3 0 1}$ & $\mathbf{0 . 0 0 9 4}$ & 0.0869 & 0.6294 \\
46 Cuneus R & 0.2178 & 0.0723 & $\mathbf{0 . 4 4 6 1}$ & $\mathbf{0 . 0 0 6 9}$ & -0.0338 & 0.8519 \\
49 Occipital Sup L & 0.2102 & 0.0831 & $\mathbf{0 . 4 4 1 7}$ & $\mathbf{0 . 0 0 7 5}$ & -0.0097 & 0.9578 \\
50 Occipital Sup R & 0.2337 & 0.0535 & $\mathbf{0 . 4 5 7 1}$ & $\mathbf{0 . 0 0 5 5}$ & -0.0267 & 0.8826 \\
51 Occipital Mid L & 0.2162 & 0.0754 & $\mathbf{0 . 4 3 0 9}$ & $\mathbf{0 . 0 0 9 2}$ & -0.0331 & 0.8548 \\
65 Angular L & 0.1127 & 0.3558 & $\mathbf{0 . 3 5 1 4}$ & $\mathbf{0 . 0 3 6 2}$ & -0.0575 & -0.7500 \\
66 Angular R & $\mathbf{0 . 1 8 5 9}$ & $\mathbf{0 . 0 4 6 4}$ & 0.2762 & 0.1031 & 0.1390 & 0.4388 \\
69 Paracentral Lobule L & $\mathbf{0 . 2 8 8 7}$ & $\mathbf{0 . 0 1 6 4}$ & 0.2046 & 0.2303 & 0.3225 & 0.0676 \\
74 Putamen R & $\mathbf{0 . 2 5 7 5}$ & $\mathbf{0 . 0 3 3 0}$ & 0.3040 & 0.0718 & 0.1922 & 0.2827 \\
79 Heschl L & 0.2288 & 0.0588 & 0.1292 & 0.4511 & $\mathbf{0 . 3 6 0 6}$ & $\mathbf{0 . 0 3 9 9}$ \\
\hline
\end{tabular}

Figures 5 and 6 show the ROIs having the biggest correlation coefficient for each analysis. We decided not to present all ROI graphics to allow a comprehensible reading. Moreover, in each graphic, the correlation line and the standard deviation are depicted. The reader should note that the correlation coefficient did not reach values higher than 0.75 . However, in our case, Pearson's coefficient was influenced by several factors, such as the number of subjects and the clinical condition, i.e., the MMSE. In fact, the distribution of the correlation coefficient values fell within the range observable in other literature surveys [74].

Finally, the SVM classification was tested. Firstly, the performance of the unimodal classification method in the identification of AD from the healthy controls was tested, based on MRI or fMRI. Specifically, when using fMRI, a unimodal classification method, it was possible to achieve a classification accuracy of $62.32 \%$, a sensitivity of $66.66 \%$, and a specificity of $58.33 \%$. On the other hand, 
when relying on MRI only, the classification of AD subjects from HC for the mono-modal method showed a classification accuracy of $50.72 \%$, a sensitivity of 30\%, and a specificity of $69.44 \%$.

As a comparison with the previous two classification methods, the combined measurements of MRI, fMRI, and MMSE were used to train the SVM classifier. For each subject, the set of 90 features coming from MRI, GMV from 90 ROIs, 90 features derived from fMRI, clustering coefficient from 90 ROIs, and one feature from MMSE, was concatenated. As can be noticed from Table 5 and Figure 7, the combination of MRI, fMRI, and MMSE measurements achieved a more accurate classification and discrimination between $\mathrm{AD}$ and HC. In this case, the method could achieve a classification accuracy of $95.65 \%$, a sensitivity of $93.93 \%$, and a specificity of $97.22 \%$.

Table 5. Comparison of different SVM classifiers' performance for diverse diagnostic data.

\begin{tabular}{cccc}
\hline Methods & Accuracy (\%) & Sensibility (\%) & Specificity (\%) \\
\hline fMRI & 62.32 & 66.66 & 58.33 \\
MRI & 50.72 & 30.02 & 69.44 \\
MRI + fMRI + MMSE & 95.65 & 93.39 & 97.22 \\
\hline
\end{tabular}

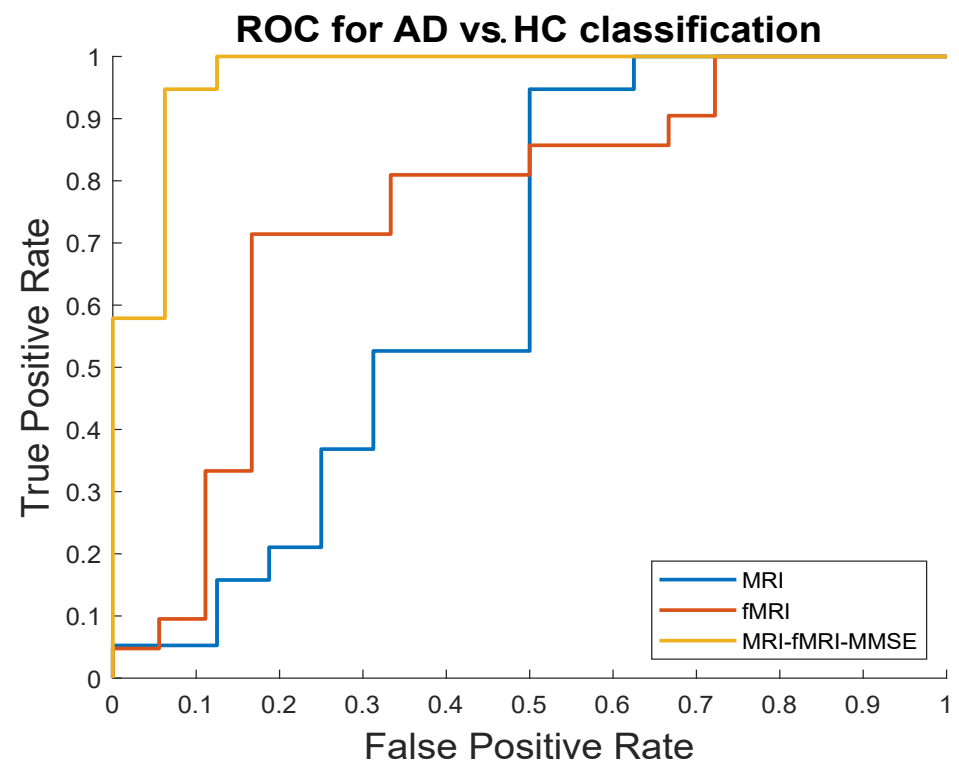

Figure 7. ROC curves of the different SVM classifiers trained using MRI, fMRI, and combined diagnostic information for recognizing AD subjects.

While other studies $[25,82]$ applied the SVM unimodal classification method or exploited images obtained from different imaging techniques [27,28], our study implemented a multimodal classification from the MRI and fMRI method combined with MMSE. The results derived from training the SVM with MRI, fMRI, and MMSE data allowed us to infer that the approach proposed in this paper is valuable and can be of potential interest to clinicians and researchers. The presented method can be adapted to carry out a similar analysis to evaluate if differences between $\mathrm{AD}$ and MCI patients exist. To this aim, the use of the MMSE as additional information and the investigation of its relationship with MRI- and fMRI-derived data can support AD research.

\section{Conclusions}

In this paper, morphological MRI and functional MRI images' processing was analyzed to verify the possibility of correlating anatomical and functional data for $\mathrm{AD}$ and $\mathrm{HC}$ subjects. First, we compared normalized whole brain volume between the HC group and AD group, which presented a lower value of this parameter. Furthermore, 36 ROIs exhibited a negative change of the GMV in AD patients compared with the HC ones. These regions are known to be involved in functions affected 
by the pathology progression $[72,83]$. Furthermore, regarding the possible correlation between GMV and cognitive status, i.e., MMSE, within groups, we had positive correlation coefficients between GMV and MMSE in left superior temporal gyrus. Our results agree with a previous study [84], where positive correlation between MMSE and a volumetric measure, i.e., volumes of deep GM, was reported. This demonstrates that pathology differences are strictly linked to a minor brain volume in AD patients and to physiological aging in HC. Successively, the potential association between GMV and CC in the ROIs was analyzed to correlate anatomical and functional results.

In fact, in the literature about AD research, to the best of our knowledge, nobody has demonstrated this promising relationship. The AD group showed a positive correlation in 4 ROIs, while in the HC group, there were nine ROIs correlated. This showed the relationship between morphological and functional data. On the other hand, this did not allow direct classification between the two groups, but only highlights a possible relationship between function and structure; hence the necessity to perform the SVM classification. In our study, we put in prominence the investigation of the differences between the AD group and HC, combining the structural, functional, and clinical features, through MRI, fMRI, and MMSE, respectively. Indeed, the unimodal classification methods, which use only one feature, showed poor accuracy and low values of sensitivity and specificity. Moreover, unlike other studies $[27,28]$, we used the fMRI technique. The use of BOLD signals and derived measurements from $\mathrm{fMRI}$ allowed a less troublesome investigation since this approach does not employ radiative contrast agents or require the acquisition of liquid biological samples $[27,28]$. Moreover, at the same time, it provides helpful information related to AD status. Moreover, while other studies $[25,82]$ used only MRI images, we proposed a multimodal method that combines MRI, fMRI, and MMSE with an increased accuracy.

Additional studies could provide further analysis and could confirm these findings. The brain volumes can be correlated with other metrics, which can be used to derive other features, e.g., the correlation of GM with path length measurements. This would further help to understand the link between structure and function in AD subjects. Instead, through the use of a classifier, it was possible to discriminate AD patients from healthy controls, and this could be a potential use in clinical applications.

Author Contributions: C.D. was the main contributor to this work. A.F. and G.M. supervised, planned, and organized the work. C.D., S.C., and M.B.L. organized the data, performed the statistical analysis, and ran the test with the classifiers. C.D., A.F., M.B.L., and G.M. wrote the original draft and edited the text.

Funding: This work was partially funded by the Artificial Intelligence in Medicine (AIM) project, granted by INFN(CSN5).

Acknowledgments: The authors thanks Matteo Fraschini (Scopus author id: 6507309070) for his valuable suggestions that helped to improve this paper. The image in the visual summary was created with the BrainNet viewer (http:/ / www.nitric.org/projects/bnv/), found in M. Xia, J. Wang, Y. He, “BrainNet Viewer: a network visualization tool for human brain connectomics", PloS one, vol. 8, No. 7, 2013, e68910 (DOI: 10.1371/journal.pone.0068910). Data collection and sharing for this project were funded by the Alzheimer's Disease Neuroimaging Initiative (ADNI) (National Institutes of Health Grant U01AG024904) and DOD ADNI (Department of Defense Award Number W81XWH-12-2e0012). ADNI is funded by the National Institute on Aging, the National Institute of Biomedical Imaging and Bioengineering, and through generous contributions from the following: Alzheimer's Association; Alzheimer's Drug Discovery Foundation; Araclon Biotech; BioClinica, Inc; Biogen Idec Inc; Bristol-Myers Squibb Company; Eisai Inc; Elan Pharmaceuticals, Inc; Eli Lilly and Company; EuroImmun; F. Hoffmann-La Roche Ltd and its affiliated company Genentech, Inc; Fujirebio; GE Healthcare; IXICO Ltd; Janssen Alzheimer Immunotherapy Research \& Development, LLC; Johnson \& Johnson Pharmaceutical Research \& Development, LLC; Medpace, Inc; Merck \& Co, Inc; Meso Scale Diagnostics, LLC; NeuroRx Research; Neurotrack Technologies; Novartis Pharmaceuticals Corporation; Pfizer, Inc; Piramal Imaging; Servier; Synarc, Inc; and Takeda Pharmaceutical Company. The CIHRis providing funds to support ADNI clinical sites in Canada. Private sector contributions are facilitated by the Foundation for the National Institutes of Health. The grantee organization is the Northern California Institute for Research and Education, and the study is coordinated by the Alzheimer's Disease Cooperative Study at the University of California, San Diego. ADNI data were disseminated by the Laboratory for Neuro Imaging at the University of Southern California. We would also like to thank all volunteers and their families for their time and commitment. Without them, ADNI could not exist. The authors wish to sincerely thank the reviewer for their comments and suggestions, which helped to enhance the quality of this paper and its clinical relevance. 
Conflicts of Interest: The authors declare no conflict of interest.

\section{Abbreviations}

The following abbreviations are used in this manuscript:

$\begin{array}{ll}\text { AD } & \text { Alzheimer's Disease } \\ \text { ADNI } & \text { Alzheimer's Disease Neuroimaging Initiative } \\ \text { ART } & \text { Artifact Detection Tools } \\ \text { BOLD } & \text { Blood Oxygenation Level Dependent } \\ \text { BCT } & \text { Brain Connectivity Toolbox } \\ \text { CC } & \text { Clustering Coefficient } \\ \text { CDR } & \text { Clinical Dementia Rating } \\ \text { CNR } & \text { Contrast-to-Noise Ratio } \\ \text { CSF } & \text { Cerebrospinal Fluid } \\ \text { dHB } & \text { Deoxyhemoglobin } \\ \text { FA } & \text { Flip Angle } \\ \text { FDG-PET } & \text { Fluorodeoxyglucose Positron Emission Tomography } \\ \text { fMRI } & \text { Functional Magnetic Resonance Imaging } \\ \text { GM } & \text { Grey Matter } \\ \text { GMM } & \text { Gaussian Mixture Model } \\ \text { GMV } & \text { Grey Matter Volume } \\ \text { HC } & \text { Health Control } \\ \text { MRI } & \text { Magnetic Resonance Imaging } \\ \text { MDI } & \text { Mild Cognitive Impairment } \\ \text { MMSE } & \text { Mini Mental State Examination } \\ \text { MNI } & \text { Montreal Neurological Institute } \\ \text { NPI-Q } & \text { Neuropsychiatric Inventor Questionnaire } \\ \text { SNR } & \text { Signal to Noise Ratio } \\ \text { SPM } & \text { Statistical Parametric Map } \\ \text { SVM } & \text { Support Vector Machine } \\ \text { TE } & \text { Time Echo } \\ \text { TR } & \text { Time Repetition } \\ \text { WM } & \text { White Matter } \\ & \end{array}$

\section{References}

1. Winter, F.; Bludszuweit-Philipp, C.; Wolkenhauer, O. Mathematical analysis of the influence of brain metabolism on the BOLD signal in Alzheimer's disease. J. Cereb. Blood Flow Metab. 2018, 38, 304-316. [CrossRef] [PubMed]

2. Xu, C.; Pham, D.L.; Rettmann, M.E.; Yu, D.N.; Prince, J.L. Reconstruction of the human cerebral cortex from magnetic resonance images. IEEE Trans. Med. Imaging 1999, 18, 467-480. [CrossRef] [PubMed]

3. Logothetis, N.K. The neural basis of the blood-oxygen-level-dependent functional magnetic resonance imaging signal. Philos. Trans. R. Soc. Lond. Ser. B Biol. Sci. 2002, 357, 1003-1037. [CrossRef]

4. Coriasco, M.; Rampado, O.; Bradac, G.B. Elements of Magnetic Resonance, in Imaging E Formation, 3rd ed.; Springer: Berlin/Heidelberg, Germany, 2014; Chapter 6.

5. Frisoni, G.B.; Fox, N.C.; Jack, C.R., Jr.; Scheltens, P.; Thompson, P.M. The clinical use of structural MRI in Alzheimer disease. Nat. Rev. Neurol. 2010, 6, 67. [CrossRef] [PubMed]

6. Thompson, P.M.; MacDonald, D.; Mega, M.S.; Holmes, C.J.; Evans, A.C.; Toga, A.W. Detection and mapping of abnormal brain structure with a probabilistic atlas of cortical surfaces. J. Comput. Assist. Tomogr. 1997, 21,567-581. [CrossRef] [PubMed]

7. Buchanan, R.W.; Vladar, K.; Barta, P.E.; Pearlson, G.D. Structural evaluation of the prefrontal cortex in schizophrenia. Am. J. Psychiatry 1998, 155, 1049-1055. [CrossRef] [PubMed]

8. Fisher, E.; Lee, J.C.; Nakamura, K.; Rudick, R.A. Gray matter atrophy in multiple sclerosis: A longitudinal study. Ann. Neurol. 2008, 64, 255-265. [CrossRef] 
9. Fox, N.C.; Freeborough, P.A. Brain atrophy progression measured from registered serial MRI: Validation and application to Alzheimer's disease. J. Magn. Reson. Imaging 1997, 7, 1069-1075. [CrossRef]

10. Munoz, D.G.; Feldman, H. Causes of Alzheimer's disease. CMAJ 2000, 162, 65-72.

11. Gur, R.E.; McGrath, C.; Chan, R.M.; Schroeder, L.; Turner, T.; Turetsky, B.I.; Kohler, C.; Alsop, D.; Maldjian, J.; Ragland, J.D.; et al. An fMRI study of facial emotion processing in patients with schizophrenia. Am. J. Psychiatry 2002, 159, 1992-1999. [CrossRef]

12. Rombouts, S.A.; Barkhof, F.; Goekoop, R.; Stam, C.J.; Scheltens, P. Altered resting state networks in mild cognitive impairment and mild Alzheimer's disease: An fMRI study. Hum. Brain Mapp. 2005, 26, 231-239. [CrossRef] [PubMed]

13. Smith, A.M.; Lewis, B.K.; Ruttimann, U.E.; Frank, Q.Y.; Sinnwell, T.M.; Yang, Y.; Duyn, J.H.; Frank, J.A. Investigation of low frequency drift in fMRI signal. Neuroimage 1999, 9, 526-533. [CrossRef] [PubMed]

14. Triantafyllou, C.; Hoge, R.D.; Krueger, G.; Wiggins, C.J.; Potthast, A.; Wiggins, G.C.; Wald, L.L. Comparison of physiological noise at $1.5 \mathrm{~T}, 3 \mathrm{~T}$ and $7 \mathrm{~T}$ and optimization of $\mathrm{fMRI}$ acquisition parameters. Neuroimage 2005, 26, 243-250. [CrossRef] [PubMed]

15. Behzadi, Y.; Restom, K.; Liau, J.; Liu, T.T. A component based noise correction method (CompCor) for BOLD and perfusion based fMRI. Neuroimage 2007, 37, 90-101. [CrossRef] [PubMed]

16. Rubinov, M.; Sporns, O. Complex network measures of brain connectivity: Uses and interpretations. Neuroimage 2010, 52, 1059-1069. [CrossRef] [PubMed]

17. Sporns, O. The human connectome: A complex network. Ann. N. Y. Acad. Sci. 2011, 1224, 109-125. [CrossRef] [PubMed]

18. Bassett, D.S.; Bullmore, E.; Verchinski, B.A.; Mattay, V.S.; Weinberger, D.R.; Meyer-Lindenberg, A. Hierarchical organization of human cortical networks in health and schizophrenia. J. Neurosci. 2008, 28, 9239-9248. [CrossRef]

19. Braun, U.; Muldoon, S.F.; Bassett, D.S. On human brain networks in health and disease. In eLS; John Wiley \& Sons, Ltd.: Chichester, UK, 2001; pp. 1-9.

20. Fox, N.C.; Cousens, S.; Scahill, R.; Harvey, R.J.; Rossor, M.N. Using serial registered brain magnetic resonance imaging to measure disease progression in Alzheimer disease: Power calculations and estimates of sample size to detect treatment effects. Arch. Neurol. 2000, 57, 339-344. [CrossRef] [PubMed]

21. Sanz-Arigita, E.J.; Schoonheim, M.M.; Damoiseaux, J.S.; Rombouts, S.A.; Maris, E.; Barkhof, F.; Scheltens, P.; Stam, C.J. Loss of 'small-world'networks in Alzheimer's disease: Graph analysis of FMRI resting-state functional connectivity. PLoS ONE 2010, 5, e13788. [CrossRef]

22. Machulda, M.M.; Ward, H.; Borowski, B.; Gunter, J.; Cha, R.; O’brien, P.; Petersen, R.C.; Boeve, B.F.; Knopman, D.; Tang-Wai, D.; et al. Comparison of memory fMRI response among normal, MCI, and Alzheimer's patients. Neurology 2003, 61, 500-506. [CrossRef]

23. He, Y.; Wang, L.; Zang, Y.; Tian, L.; Zhang, X.; Li, K.; Jiang, T. Regional coherence changes in the early stages of Alzheimer's disease: A combined structural and resting-state functional MRI study. Neuroimage 2007, 35, 488-500. [CrossRef]

24. Calamante, F.; Smith, R.E.; Liang, X.; Zalesky, A.; Connelly, A. Track-weighted dynamic functional connectivity (TW-dFC): A new method to study time-resolved functional connectivity. Brain Struct. Funct. 2017, 222, 3761-3774. [CrossRef] [PubMed]

25. Khedher, L.; Ramírez, J.; Górriz, J.M.; Brahim, A.; Segovia, F.; Alzheimer's Disease Neuroimaging Initiative. Early diagnosis of Alzheimer's disease based on partial least squares, principal component analysis and support vector machine using segmented MRI images. Neurocomputing 2015, 151, 139-150. [CrossRef]

26. Abdulkadir, A.; Peter, J.; Ronneberger, O.; Brox, T.; Klöppel, S. Voxel-based multi-class classification of $\mathrm{AD}, \mathrm{MCI}$, and elderly controls. In Proceedings of the Medical Image Computing and Computer-Assisted Intervention (MICCAI) 2014 CADDementia Challenge, Boston, MA, USA, 14-18 September 2014; pp. 1-8.

27. Dukart, J.; Mueller, K.; Barthel, H.; Villringer, A.; Sabri, O.; Schroeter, M.L.; Alzheimer's Disease Neuroimaging Initiative. Meta-analysis based SVM classification enables accurate detection of Alzheimer's disease across different clinical centers using FDG-PET and MRI. Psychiatry Res. Neuroimaging 2013, 212, 230-236. [CrossRef]

28. Zhang, D.; Wang, Y.; Zhou, L.; Yuan, H.; Shen, D.; Alzheimer's Disease Neuroimaging Initiative. Multimodal classification of Alzheimer's disease and mild cognitive impairment. Neuroimage 2011, 55, 856-867. [CrossRef] [PubMed] 
29. Kaufer, D.I.; Cummings, J.L.; Ketchel, P.; Smith, V.; MacMillan, A.; Shelley, T.; Lopez, O.L.; DeKosky, S.T. Validation of the NPI-Q, a brief clinical form of the Neuropsychiatric Inventory. J. Neuropsychiatry Clin. Neurosci. 2000, 12, 233-239. [CrossRef]

30. Morris, J.C. Clinical dementia rating: A reliable and valid diagnostic and staging measure for dementia of the Alzheimer type. Int. Psychogeriatr. 1997, 9, 173-176. [CrossRef]

31. Nieuwenhuis-Mark, R.E. The death knoll for the MMSE: Has it outlived its purpose? J. Geriatr. Psychiatry Neurol. 2010, 23, 151-157. [CrossRef]

32. Uğurbil, K. Magnetic resonance imaging at ultrahigh fields. IEEE Trans. Biomed. Eng. 2014, 61, 1364-1379. [CrossRef]

33. Ballester, M.A.G.; Zisserman, A.P.; Brady, M. Estimation of the partial volume effect in MRI. Med. Image Anal. 2002, 6, 389-405.

34. Weishaupt, D.; Köchli, V.D.; Marincek, B. How Does MRI Work? An Introduction to the Physics and Function of Magnetic Resonance Imaging; Springer Science \& Business Media: Berlin/Heidelberg, Germany, 2008.

35. Pohmann, R.; Speck, O.; Scheffler, K. Signal-to-noise ratio and MR tissue parameters in human brain imaging at 3, 7, and 9.4 tesla using current receive coil arrays. Magn. Reson. Med. 2016, 75, 801-809. [CrossRef]

36. Hyder, F.; Kida, I.; Behar, K.L.; Kennan, R.P.; Maciejewski, P.K.; Rothman, D.L. Quantitative functional imaging of the brain: towards mapping neuronal activity by BOLD fMRI. NMR Biomed. 2001, 14, 413-431. [CrossRef]

37. Whitfield-Gabrieli, S.; Nieto-Castanon, A. Conn: A functional connectivity toolbox for correlated and anticorrelated brain networks. Brain Connect. 2012, 2, 125-141. [CrossRef]

38. Gili, T.; Cercignani, M.; Serra, L.; Perri, R.; Giove, F.; Maraviglia, B.; Caltagirone, C.; Bozzali, M. Regional brain atrophy and functional disconnection across Alzheimer's disease evolution. J. Neurol. Neurosurg. Psychiatry 2011, 82, 58-66. [CrossRef]

39. Cuingnet, R.; Gerardin, E.; Tessieras, J.; Auzias, G.; Lehéricy, S.; Habert, M.O.; Chupin, M.; Benali, H.; Colliot, O.; Initiative, A.D.N.; et al. Automatic classification of patients with Alzheimer's disease from structural MRI: A comparison of ten methods using the ADNI database. Neuroimage 2011, 56, 766-781. [CrossRef]

40. Klöppel, S.; Stonnington, C.M.; Chu, C.; Draganski, B.; Scahill, R.I.; Rohrer, J.D.; Fox, N.C.; Jack, C.R., Jr.; Ashburner, J.; Frackowiak, R.S. Automatic classification of MR scans in Alzheimer's disease. Brain 2008, 131, 681-689. [CrossRef]

41. Bron, E.E.; Smits, M.; Van Der Flier, W.M.; Vrenken, H.; Barkhof, F.; Scheltens, P.; Papma, J.M.; Steketee, R.M.; Orellana, C.M.; Meijboom, R.; et al. Standardized evaluation of algorithms for computer-aided diagnosis of dementia based on structural MRI: The CADDementia challenge. NeuroImage 2015, 111, 562-579. [CrossRef]

42. Khazaee, A.; Ebrahimzadeh, A.; Babajani-Feremi, A. Identifying patients with Alzheimer's disease using resting-state fMRI and graph theory. Clin. Neurophysiol. 2015, 126, 2132-2141. [CrossRef]

43. Larobina, M.; Murino, L. Medical image file formats. J. Digit. Imaging 2014, 27, 200-206. [CrossRef]

44. Challis, J.H. A procedure for determining rigid body transformation parameters. J. Biomech. 1995, $28,733-737$. [CrossRef]

45. Tzourio-Mazoyer, N.; Landeau, B.; Papathanassiou, D.; Crivello, F.; Etard, O.; Delcroix, N.; Mazoyer, B.; Joliot, M. Automated anatomical labeling of activations in SPM using a macroscopic anatomical parcellation of the MNI MRI single-subject brain. Neuroimage 2002, 15, 273-289. [CrossRef]

46. Sladky, R.; Friston, K.J.; Tröstl, J.; Cunnington, R.; Moser, E.; Windischberger, C. Slice-timing effects and their correction in functional MRI. Neuroimage 2011, 58, 588-594. [CrossRef]

47. Hellier, P.; Ashburner, J.; Corouge, I.; Barillot, C.; Friston, K.J. Inter-subject registration of functional and anatomical data using SPM. In International Conference on Medical Image Computing and Computer-Assisted Intervention; Springer: Berlin/Heidelberg, Germany, 2002; pp. 590-597.

48. Despotović, I.; Goossens, B.; Philips, W. MRI segmentation of the human brain: Challenges, methods, and applications. Comput. Math. Methods Med. 2015, 2015, 450341. [CrossRef]

49. Penny, W.D.; Friston, K.J.; Ashburner, J.T.; Kiebel, S.J.; Nichols, T.E. Statistical Parametric Mapping: The Analysis of Functional Brain Images; Elsevier: Amsterdam, The Netherlands, 2011.

50. Ashburner, J.; Friston, K.J. Unified segmentation. Neuroimage 2005, 26, 839-851. [CrossRef] 
51. Solé-Padullés, C.; Bartrés-Faz, D.; Junqué, C.; Vendrell, P.; Rami, L.; Clemente, I.C.; Bosch, B.; Villar, A.; Bargalló, N.; Jurado, M.A.; et al. Brain structure and function related to cognitive reserve variables in normal aging, mild cognitive impairment and Alzheimer's disease. Neurobiol. Aging 2009, 30, 1114-1124. [CrossRef]

52. Coffey, C.E.; Lucke, J.F.; Saxton, J.A.; Ratcliff, G.; Unitas, L.J.; Billig, B.; Bryan, R.N. Sex differences in brain aging: A quantitative magnetic resonance imaging study. Arch. Neurol. 1998, 55, 169-179. [CrossRef]

53. Maldjian, J.A.; Laurienti, P.J.; Kraft, R.A.; Burdette, J.H. An automated method for neuroanatomic and cytoarchitectonic atlas-based interrogation of fMRI data sets. Neuroimage 2003, 19, 1233-1239. [CrossRef]

54. Magnin B.; Mesrob L.; Kinkingnehun S.; Pelegrini-Issac M.; Colliot O.; Sarazin M.; Dubois B.; Lehericy S.; Benali H. Support vector machine-based classification of Alzheimer's disease from whole-brain anatomical MRI. Neuroradiology 2009, 51, 73-83. [CrossRef]

55. Amico, F.; Stauber, J.; Koutsouleris, N.; Frodl, T. Anterior cingulate cortex gray matter abnormalities in adults with attention deficit hyperactivity disorder: A voxel-based morphometry study. Psychiatry Res. Neuroimaging 2011, 191, 31-35. [CrossRef]

56. Tijms, B.M.; Wink, A.M.; de Haan, W.; van der Flier, W.M.; Stam, C.J.; Scheltens, P.; Barkhof, F. Alzheimer's disease: Connecting findings from graph theoretical studies of brain networks. Neurobiol. Aging 2013, 34, 2023-2036. [CrossRef]

57. Bourisly, A.K.; Gejo, G.; Hayat, A.A.; Alsarraf, L.; Dashti, F.M.; Di Paola, M. White matter sexual dimorphism of the adult human brain. Transl. Neurosci. 2017, 8, 49-53. [CrossRef]

58. Wolk, D.A.; Price, J.C.; Saxton, J.A.; Snitz, B.E.; James, J.A.; Lopez, O.L.; Aizenstein, H.J.; Cohen, A.D.; Weissfeld, L.A.; Mathis, C.A.; et al. Amyloid imaging in mild cognitive impairment subtypes. Ann. Neurol. 2009, 65, 557-568. [CrossRef]

59. Friston, K.J. Functional and effective connectivity in neuroimaging: A synthesis. Hum. Brain Mapp. 1994, 2, 56-78. [CrossRef]

60. Liang, X.; Wang, J.; Yan, C.; Shu, N.; Xu, K.; Gong, G.; He, Y. Effects of different correlation metrics and preprocessing factors on small-world brain functional networks: A resting-state functional MRI study. PLoS ONE 2012, 7, e32766. [CrossRef]

61. Supekar, K.; Menon, V.; Rubin, D.; Musen, M.; Greicius, M.D. Network analysis of intrinsic functional brain connectivity in Alzheimer's disease. PLoS Comput. Biol. 2008, 4, e1000100. [CrossRef]

62. Stam, C.; De Haan, W.; Daffertshofer, A.; Jones, B.; Manshanden, I.; van Cappellen van Walsum, A.M.; Montez, T.; Verbunt, J.; De Munck, J.; Van Dijk, B.; et al. Graph theoretical analysis of magnetoencephalographic functional connectivity in Alzheimer's disease. Brain 2008, 132, 213-224. [CrossRef]

63. Yao, Z.; Zhang, Y.; Lin, L.; Zhou, Y.; Xu, C.; Jiang, T.; Alzheimer's Disease Neuroimaging Initiative. Abnormal cortical networks in mild cognitive impairment and Alzheimer's disease. PLoS Comput. Biol. 2010, 6, e1001006. [CrossRef]

64. Stam, C.J.; Reijneveld, J.C. Graph theoretical analysis of complex networks in the brain. Nonlinear Biomed. Phys. 2007, 1, 3. [CrossRef]

65. Abdi, H. Bonferroni and Šidák corrections for multiple comparisons. Encycl. Meas. Stat. 2007, 3, $103-107$.

66. Verhoeven, K.J.; Simonsen, K.L.; McIntyre, L.M. Implementing false discovery rate control: Increasing your power. Oikos 2005, 108, 643-647. [CrossRef]

67. Mavroforakis, M.E.; Theodoridis, S. A geometric approach to support vector machine (SVM) classification. IEEE Trans. Neural Netw. 2006, 17, 671-682. [CrossRef]

68. Zhou, K.; He, W.; Xu, Y.; Xiong, G.; Cai, J. Feature selection and transfer learning for Alzheimer's disease clinical diagnosis. Appl. Sci. 2018, 8, 1372. [CrossRef]

69. Cawley, G.C. Leave-one-out cross-validation based model selection criteria for weighted LS-SVMs. In Proceedings of the 2006 IEEE International Joint Conference on Neural Network, Vancouver, BC, Canada, 16-21 July 2006; pp. 1661-1668.

70. Tombaugh, T.; McDowell, I.; Kristjansson, B.; Hubley, A. Mini-Mental State Examination (MMSE) and the Modified MMSE (3MS): A psychometric comparison and normative data. Psychol. Assess. 1996, 8, 48. [CrossRef]

71. Ruxton, G.D. The unequal variance t-test is an underused alternative to Student's t-test and the Mann-Whitney U test. Behav. Ecol. 2006, 17, 688-690. [CrossRef]

72. Resnick, S.M.; Goldszal, A.F.; Davatzikos, C.; Golski, S.; Kraut, M.A.; Metter, E.J.; Bryan, R.N.; Zonderman, A.B. One-year age changes in MRI brain volumes in older adults. Cereb. Cortex 2000, 10, 464-472. [CrossRef] 
73. Hafkemeijer, A.; Möller, C.; Dopper, E.G.; Jiskoot, L.C.; Schouten, T.M.; van Swieten, J.C.; van der Flier, W.M.; Vrenken, H.; Pijnenburg, Y.A.; Barkhof, F.; et al. Resting state functional connectivity differences between behavioral variant frontotemporal dementia and Alzheimer's disease. Front. Hum. Neurosci. 2015, 9, 474. [CrossRef]

74. Song, J.; Qin, W.; Liu, Y.; Duan, Y.; Liu, J.; He, X.; Li, K.; Zhang, X.; Jiang, T.; Yu, C. Aberrant functional organization within and between resting-state networks in AD. PLoS ONE 2013, 8, e63727. [CrossRef]

75. Kim, J.; Kim, Y.H.; Lee, J.H. Hippocampus-precuneus functional connectivity as an early sign of Alzheimer's disease: A preliminary study using structural and functional magnetic resonance imaging data. Brain Res. 2013, 1495, 18-29. [CrossRef]

76. Rémy, F.; Mirrashed, F.; Campbell, B.; Richter, W. Verbal episodic memory impairment in Alzheimer's disease: A combined structural and functional MRI study. Neuroimage 2005, 25, 253-266. [CrossRef]

77. He, X.; Qin, W.; Liu, Y.; Zhang, X.; Duan, Y.; Song, J.; Li, K.; Jiang, T.; Yu, C. Abnormal salience network in normal aging and in amnestic mild cognitive impairment and Alzheimer's disease. Hum. Brain Mapp. 2014, 35, 3446-3464. [CrossRef]

78. Folstein, M.F.; Folstein, S.E.; McHugh, P.R. "Mini-mental state": A practical method for grading the cognitive state of patients for the clinician. J. Psychiatr. Res. 1975, 12, 189-198. [CrossRef]

79. Mukaka, M.M. A guide to appropriate use of correlation coefficient in medical research. Malawi Med. J. 2012, 24, 69-71.

80. Douaud, G.; Refsum, H.; de Jager, C.A.; Jacoby, R.; Nichols, T.E.; Smith, S.M.; Smith, A.D. Preventing Alzheimer's disease-related gray matter atrophy by B-vitamin treatment. Proc. Natl. Acad. Sci. USA 2013, 110, 9523-9528. [CrossRef]

81. Wang, Z.; Yan, C.; Zhao, C.; Qi, Z.; Zhou, W.; Lu, J.; He, Y.; Li, K. Spatial patterns of intrinsic brain activity in mild cognitive impairment and alzheimer's disease: A resting-state functional MRI study. Hum. Brain Mapp. 2011, 32, 1720-1740. [CrossRef]

82. Yang, W.; Lui, R.L.; Gao, J.H.; Chan, T.F.; Yau, S.T.; Sperling, R.A.; Huang, X. Independent component analysis-based classification of Alzheimer's disease MRI data. J. Alzheimer's Dis. 2011, 24, 775-783. [CrossRef]

83. Mueller, E.; Moore, M.; Kerr, D.; Sexton, G.; Camicioli, R.; Howieson, D.; Quinn, J.; Kaye, J.A. Brain volume preserved in healthy elderly through the eleventh decade. Neurology 1998, 51, 1555-1562. [CrossRef]

84. de Jong, L.W.; van der Hiele, K.; Veer, I.M.; Houwing, J.; Westendorp, R.; Bollen, E.; de Bruin, P.W.; Middelkoop, H.; van Buchem, M.A.; van der Grond, J. Strongly reduced volumes of putamen and thalamus in Alzheimer's disease: An MRI study. Brain 2008, 131, 3277-3285. [CrossRef]

(C) 2019 by the authors. Licensee MDPI, Basel, Switzerland. This article is an open access article distributed under the terms and conditions of the Creative Commons Attribution (CC BY) license (http:/ / creativecommons.org/licenses/by/4.0/). 